\title{
Role of Macrophages in Vascular Regeneration
}

\author{
Shirin Saberianpour (iD ${ }^{1, *}$ \\ ${ }^{1}$ Vascular and Endovascular Surgery Research Center, Mashhad University of Medical Sciences, Mashhad, Iran \\ "Corresponding author: Vascular and Endovascular Surgery Research Center, Mashhad University of Medical Sciences, Mashhad, Iran. Email: saberianpoursh@mums.ac.ir
}

Received 2020 June 13; Accepted 2020 October 17.

\begin{abstract}
Angiogenesis is very important for most strategies for regenerative damaged organs. The natural inflammatory response can be affected by the activity of different types of macrophages and their cytokines. Macrophages exist in a wide variety of phenotypes. These macrophages can range from classic M1 to M2 macrophages. M2 macrophages themselves can include different types of subgroups. These types of macrophages are usually effective in vascular regeneration while M1 or classic macrophages play a role in chronic wounds. The macrophages move toward the M2 phenotype or inhibit the polarization of the macrophages toward the M1 phenotype. On the other hand, macrophage polarization can be affected by a variety of factors, such as different types of miRNAs. The process of vascular repair and enhancing the therapeutic potential of drug regulators the treatment of vascular damage through modification of macrophage polarization. Macrophages do this by various mechanisms, which are described in detail in this study. Finally, new therapeutic mechanisms aimed at macrophages in vascular disease are discussed.
\end{abstract}

Keywords: Macrophage, Vascular, Angiogenesis, Regeneration

\section{Context}

Inflammation is a complex process in which cells and various factors in a certain way protect tissues against various injuries. In fact, inflammation is a controlled outcome between inflammatory and anti-inflammatory factors during which the activity, proliferation, and chemotaxis of cells are regulated. Proliferation has a large number of inflammatory cells, blood vessels created by vasodilation, and a number of inflammatory mediators. There is also an area of damaged tissue that is deficient in relative oxygen, which is associated with vascular growth. In the process of inflammation, a set of cells and chemical agents are involved. In addition to involving endothelial cells, the angiogenesis process also involves other cells, including inflammatory cells. Among the cells, macrophages are very important. These cells are involved in the process of angiogenesis due to growth factors. These factors connect leukocytes to vascular endothelium and initiate the repair process. During injury, monocytes guide a number of inflammatory mediators and chemical agents to migrate to the affected area to activate macrophages, inflammatory agents and a number of R Factors. They help to repair the environment by causing damage to cells such as the endothelium, epithelium, or mesenchymal. They provide the conditions for tissue repair and angiogenesis. The role of macrophages in changing the polarization of these cells in improving angiogenesis has been described $(1,2)$.

\section{Macrophage Phenotypic Diversity}

Due to their phenotypic diversity, macrophages also show different functions in homeostasis. Different types of macrophages play a key role in guiding the immune response at different physiological stages, including growth, injury, and chronic inflammation. In addition, tissueresident macrophages in many organs have unique gene profiles (3). And it performs the specific tasks necessary for a healthy body function (4). Significant advances in the design of specialized cellular diagnostic techniques have led to the specialized diagnosis of immune cell phenotypes, including macrophages. This leads to a deeper understanding of the different phenotypes of macrophages. Their origin and phenotype should be discussed $(4,5)$.

Macrophages can form different types of phenotypes under different factors. M1 phenotypes are usually formed under the influence of different cytokines such as IFN- $\gamma$, TNF- $\alpha$ and LPS to M1 phenotype, and in contrast to M2 macrophages which are formed under the influence of IL4 cytokines and IL-10. These anti-inflammatory cytokines improve the angiogenesis process. In terms of metabolic pathways, M1 macrophages obtain energy from glycolysis and arginine pathways. M2 macrophages obtain their en- 
ergy through oxidative pathways. M2s are classified according to their broad classification. For example, M2C usually represents inflammatory macrophages that suppress inflammatory cytokines (6-12).

\section{Angiogenesis/Vascularization}

Blood vessels deliver oxygen, metabolites, export wastes, and the formation of new blood vessels that is essential to improve tissue function after ischemia or tissue damage $(13,14)$.vascular function is undeniably associated with the preservation and repair of the damaged vascular (14). Endothelial precursor cells (EPCs) are involved in newly formed capillaries and stimulate ischemic tissue vessels (15). Endothelial precursor cells are transported from the bone marrow to the site of vascular injury, playing an important role in postoperative vascular repair. Biomechanical tracking Research shows that bone marrow-derived cells with EPC cell markers play an important role in vascular healing (16). One of the advantages of using EPC in vascular healing is the physical formation of new capillaries, which regulate processes such as angiogenesis during inflammation and recovery with the effect of paracrine (17). Like fat-producing hematopoietic cells, bone marrow, cells of mesenchymal origin, paracrine VEGF factors such as increase angiogenesis (18).

\section{The General Role of Macrophages in Regeneration}

There is considerable evidence that macrophages are key regulators of the angiogenesis process. They play different roles to ensure proper regeneration (19). It is established that the macrophage phenotype evolves the angiogenesis process (20). In an acute injury, with the onset of tissue repair, a large number of macrophage populations are transmitted to the injured place (21). Endothelial cells restore the vessels. This process eventually results in angiogenesis repair and scarring (22). Recently, an important molecular mechanism has been discovered in M2 macrophages that promotes angiogenesis, permeability, and the absorption of inflammatory cells in various human diseases. It can initiate angiogenesis from various mechanisms. This process itself causes HIF1 $\alpha$ and VEGF-A $(23,24)$.

\section{Paracrine Roles of Types of Macrophages in Angio- genesis}

Macrophages secrete a variety of growth factors associated with angiogenesis (12). The secretion of VEGF-A by macrophages is important for the stimulation of angiogenesis in the repair of damaged tissues. Also, the polarization of macrophages will cause the secretion of growth factors that can again affect the angiogenesis process (25). By analyzing gene expression and angiocrine secretion profiles from different macrophage phenotypes, it was shown that M1 macrophages expressed genes involved in the onset of angiogenesis, including VEGF-A and FGF2 $(12,26)$ Studies have shown that M2a macrophages are able to secrete high levels of PDGF factor, while M2c macrophages secrete high levels of MMP-9, according to a study that analyzed macrophages within collagen scaffolds. It was shown in mice that different phenotypes were involved in the angiogenesis process $(12,27)$. Collagen scaffolding with macrophages can reduce the strength of angiogenesis because collagen scaffolding can act as a scaffold around the M2 macrophage to prevent it from working (28). In contrast, glutaraldehyde collagen scaffolds improve angiogenesis by using both M1 and M2 macrophages (29). M1 macrophages play a role in stimulating capillary germination, while M2 macrophages help stabilize arteries (30). Strategies for controlling macrophage dynamics may achieve more effective angiogenesis (30). In one study, concomitant delivery of CSF1 with VEGF-A in hydrogel implants resulted in stronger angiogenesis than VEGF-A alone (31). Therefore, it is important to consider which type of cell is in a ruler. Dynamic growth regulation and regeneration and how different cell types work together are important for angiogenesis process (32).

\section{Macrophage Interactions with Other Cells for En- hancement of Angiogenesis}

Macrophages with a number of other cells including endothelial cells, pericytes, and vascular smooth muscle cells are involved with inactive cells in cell-cell interactions $(33,34)$. These interactions regulate angiogenesis during embryonic development and in response to injury. Macrophage depletion in injured tissue leads to impaired vascular patterns that can be due to insufficient vascular repair. Phagocyte ability of macrophages has been shown to orient cell and cell communication with endothelial cells, which can be a bridge between two or more angiogenic buds $(35,36)$. In studies of the brain, brain cells and mouse development macrophage were connected with endothelial cells fusion $(37,38)$. Macrophages also influence the behaviors of vascular smooth muscle cells that are associated with the growth and remodeling of microcirculation (38). Recent studies have shown Macrophage polarization can be a good therapeutic goal to enhance repair in ischemic tissue areas (39). 


\section{Macrophage Differentiation Into Vascular Cells}

Macrophages may be able to differentiate vascular wall cells, including vascular smooth muscle cells, peritonitis, and endothelial cells (40). Smooth vascular muscle cells that inhabit the middle layer of large blood vessels can respond to damage in macrophages and mesenchymal stem cells (41). Macrophages are able to differentiate macrophages into SMCs (42). In the regulation of tumor angiogenesis, bone marrow-derived cells have been shown to be differentiated both in the peripheral environment and in macrophages. Distinguishing macrophages from peritonitis or macrophages to fibroblasts can have important implications for regulating angiogenesis fibrosis is an important factor (43). The expression of endothelial markers characteristic of CD31, VEGFR2, by a subset of monocytes, called myeloid vascular cells (MACs), provides another explanation for the observed phenotypic overlap (44). While MAC has been shown to play an angiogenic role and could serve as an alternative to angiogenesis M2 macrophages, their inability to differentiate into endothelial cells is a drawback to their use $(45,46)$.

\section{Exosome-Guided Phenotypic Switch of M1 to M2 Macrophages for Angiogenesis}

A method of induction of macrophage polarization is the use of exosomes. Using exosomes has been proposed to directly convert M to M2 for effective angiogenesis. Preinflammatory M1 macrophages release a wide range of preinflammatory exosomes after damage (47). M1-Exosome exerts an anti-vascular effect. They also showed highly inflammatory miRNAs such as miR-155. miR-155 can be transferred to endothelial cells (ECS) and lead to inhibition of angiogenesis by regulating genes at reducing the angiogenic ability of ECs (48). Recent studies suggest that exosomes secreted by mesenchymal stem cells may be a new mechanism for the beneficial effect of MSCs transplantation after myocardial infarction. Studies showed that MSC-Exosome impairs myocardial in mice through miR182 shuttles, which reduces the induction of macrophage polarization. Another study showed a new way to use MSCExosome as a potential treatment tool for angiogenesis $(49,50)$.

\section{9. miRNA-Mediated Macrophage Polarization}

Non-coding miRNAs are small molecules' that can regulate gene expression and network cellular processes (51). The gene and cellular regulators associated with miRNA in macrophage polarization were developed using microarray and RT-Qpcr techniques. miRNAs that modulate the polarity of macrophages may have the potential to treat inflammatory diseases. Recent findings in the expression of miRNA expression in polar macrophages of mice and human resources and a summary of how these miRNAs are regulated polarization of macrophages (Table 1$)(52,53)$.

\begin{tabular}{ll}
\hline $\begin{array}{l}\text { Table 1. lists some of these miRNAs } \\
\text { Polarization }\end{array}$ & miRNA \\
\hline M1 & $\begin{array}{l}\text { miR-181a, miR-204-5p, miR-451, miR-125a-3p, miR-125a-3p, } \\
\text { miR-155, miR-127, miR-155, miR-125b, miR-9, miR-155-5p }\end{array}$ \\
M2 & $\begin{array}{l}\text { miR-125-5p, miR-125a-5p, miR-223, miR-146a, miR-132, MiR-34a, } \\
\text { miR-146a-3p, miR-125a-5p, miR-124 }\end{array}$ \\
\hline
\end{tabular}

\section{Signaling Pathway in Angiogenesis by Macrophages}

Studies have shown that both macrophages and proteins secreted in the Wnt signaling pathway regulate angiogenesis. Therefore, according to observations, some macrophages mediate the effects of angiogenesis, and in fact, macrophages represent Wnt ligands (54). Interferon IFN- $\gamma$ and lipopolysaccharide (LPS), two potent inflammatory agents known to regulate Wnt5a protein levels in macrophages. In addition, Wnt5a can automatically control regenerative signaling $(55,56)$. Given that Wnt5a inhibits the expression of several inflammatory cytokines in endothelial cells, including IL-6 and IL-8, the Wnt5a factor has a direct effect on endothelial cell proliferation, migration, and Tie-2 expression (57). It is therefore assumed that Wnt5a, derived from macrophages, regulates angiogenesis through two direct and indirect mechanisms at different levels. In addition, Wnt5a has also been shown to regenerate macrophage chemotherapy protein, CCL2, in endothelial cells. It is possible that macrophage-derived Wnt5a could be indirectly conductive. Macrophages are involved in these vessels and the genetic approaches involved reduce the risks of macrophages that can destroy the regeneration and persistence of these vessels after delivery. Further investigation showed that the secretion of conventional Wnt protein macrophages, Wnt7b, is necessary for this process. In particular, the secretion of Ang-2 from the peritonitis causes Wnt7b expression in macrophages $(58,59)$. Wnt7b can stimulate the entry of vascular EC into phase $S$ of the cell cycle (58). Angiopoietin-2 secretion inhibits Angiopoietin-1-mediated survival signaling in the endothelial cell, as well as $\beta$-catenin signaling to induce cell cycle entry and subsequent apoptosis (60). 


\section{Macrophages as a Potential Target for Vascular Dis- ease}

Different therapeutic agent, which has been used in the generation, is anticipated to help researchers solve more clinical problems. A number of these therapeutic agents are performed to improve vascular disease and with the aim of macrophages. A number of these factors can help polarize macrophages in the position of vascular damage. The following table examines a number of these factors and therapeutic mechanisms (Table 2).

\begin{tabular}{llc}
\hline Table 2. A number of Vascular Diseases Aimed at Macrophages & \\
\hline Defection & Mechanism & References \\
\hline Chronic kidney disease & VEGF therapy was largely & (61) \\
(CKD) & driven by modulation of & \\
& renal macrophages toward & \\
& VEGF-expressing M2 & \\
& phenotype
\end{tabular}

\begin{tabular}{|l|l}
\hline Intimal hyperplasia & $\begin{array}{l}\text { Decreasing the miR-150 } \\
\text { increases the high ratio of } \\
\text { macrophages to M1 to M2, } \\
\text { which in turn will cause } \\
\text { hyperplasia. }\end{array}$ \\
\hline Atherosclerosis & $\begin{array}{l}\text { Different macrophage } \\
\text { phenotypes have different } \\
\text { effects on plaque progress. }\end{array}$ \\
\hline Wounds & $\begin{array}{l}\text { Mesenchymal cells can } \\
\text { stimulate the functional } \\
\text { repair of vascular } \\
\text { endothelial cells by } \\
\text { regenerating macrophage } \\
\text { phenotypes }\end{array}$ \\
\hline Peripheral artery disease & $\begin{array}{l}\text { Increased expression of } \\
\text { VEGF165b in macrophages } \\
\text { causes a M1-like phenotype } \\
\text { that directly interferes with } \\
\text { angiogenesis. }\end{array}$ \\
\hline Intracranial aneurysms & $\begin{array}{l}\text { Montelukast induces M2 } \\
\text { macrophage polarization } \\
\text { and prevents AAA formation }\end{array}$ \\
\hline Abdominal aortic \\
aneurysm (AAA) \\
Choroid
\end{tabular}

\section{Conclusions}

In recent years, studies on the role of macrophages in the process of many injuries have been studied. One of the most important aspects is to study the polarization of macrophages in the face of various physiological situations. The polarization of macrophages can be affected by internal or external factors. Macrophages themselves can control repair processes by using their own function or by secreting extracellular material. Macrophages play a major role in the repair of vascular structures. It can occur during vascular disease by modifying macrophage polarization and factor secretion.

\section{Acknowledgments}

The authors wish to thank the personnel of Vascular and endovascular surgery research center, Mashhad University of Medical Science.

\section{Footnotes}

Conflict of Interests: Author declare no competing financial interests exist.

Funding/Support: Vascular and endovascular surgery research center, Mashhad university of medical science, Mashhad, Iran.

\section{References}

1. Gordon S, Plüddemann A, Martinez Estrada F. Macrophage heterogeneity in tissues: phenotypic diversity and functions. Immunol Rev. 2014;262(1):36-55. doi: 10.1111/imr.12223.

2. Wynn TA, Vannella KM. Macrophages in Tissue Repair, Regeneration, and Fibrosis. Immunity. 2016;44(3):450-62. doi: 10.1016/j.immuni.2016.02.015.

3. Chaussabel D, Semnani RT, McDowell MA, Sacks D, Sher A, Nutman TB. Unique gene expression profiles of human macrophages and dendritic cells to phylogenetically distinct parasites. Blood. 2003;102(2):672-81. doi: 10.1182/blood-2002-10-3232.

4. Guo L, Akahori H, Harari E, Smith SL, Polavarapu R, Karmali V, et al. CD163+ macrophages promote angiogenesis and vascular permeability accompanied by inflammation in atherosclerosis. J Clin Invest. 2018;128(3):1106-24. doi: 10.1172/jci93025.

5. Ramirez-Pedraza M, Fernández M. Interplay Between Macrophages and Angiogenesis: A Double-Edged Sword in Liver Disease. Front Immunol. 2019;10. doi: 10.3389/fimmu.2019.02882.

6. Thomas L, Rao Z, Gerstmeier J, Raasch M, Weinigel C, Rummler S, et al. Selective upregulation of TNF $\alpha$ expression in classically-activated human monocyte-derived macrophages (M1) through pharmacological interference with V-ATPase. Biochem Pharmacol. 2017;130:71-82. doi: 10.1016/j.bcp.2017.02.004.

7. Lin L, Gao Z, Lin Y, Zhu X, Liu W, Liu D, et al. Akt, mTOR and NF- $\kappa$ B pathway activation in Treponema pallidum stimulates M1 macrophages. Int Immunopharmacol. 2018;59:181-6. doi: 10.1016/j.intimp.2018.03.040.

8. $\mathrm{Hu} \mathrm{X}$, Wang $\mathrm{H}$, Han $\mathrm{C}$, Cao X. Src promotes anti-inflammatory (M2) macrophage generation via the IL-4/STAT6 pathway. Cytokine. 2018;111:209-15. doi:10.1016/j.cyto.2018.08.030.

9. Orihuela R, McPherson CA, Harry GJ. Microglial M1/M2 polarization and metabolic states. Brit J Pharmacol. 2016;173(4):649-65. doi: 10.1111/bph.13139.

10. May RC, Lefèvre L, Galès A, Olagnier D, Bernad J, Perez L, et al. PPAR Ligands Switched High Fat Diet-Induced Macrophage M2b Polarization toward M2a Thereby Improving Intestinal Candida Elimination. PLOS ONE. 2010;5(9). e12828. doi: 10.1371/journal.pone.0012828. 
11. Lisi L, Stigliano E, Lauriola L, Navarra P, Russo CD. ProinflammatoryActivated Glioma Cells Induce a Switch in Microglial Polarization and Activation Status, From a Predominant M2b Phenotype to a Mixture of M1 and M2a/B Polarized Cells. ASN Neuro. 2014;6(3):AN20130045. doi: 10.1042/an20130045.

12. Jetten N, Verbruggen S, Gijbels MJ, Post MJ, De Winther MP, Donners MM. Anti-inflammatory M2, but not pro-inflammatory M1 macrophages promote angiogenesis in vivo. Angiogenesis. 2013;17(1):109-18. doi: 10.1007/s10456-013-9381-6.

13. Oudega M. Molecular and cellular mechanisms underlying the role of blood vessels in spinal cord injury and repair. Cell Tissue Res. 2012;349(1):269-88. doi: 10.1007/s00441-012-1440-6.

14. Yang J. The role of reactive oxygen species in angiogenesis and preventing tissue injury after brain ischemia. Microvasc Res. 2019;123:627. doi: 10.1016/j.mvr.2018.12.005.

15. Kaushik K, Das A. Endothelial progenitor cell therapy for chronic wound tissue regeneration. Cytotherapy. 2019;21(11):1137-50. doi: 10.1016/j.jcyt.2019.09.002.

16. Berezin AE. Endogenous vascular repair system in cardiovascular disease: The role of endothelial progenitor cells. Austral Med J.2019;12(2). doi:10.21767/amj.2018.3464.

17. Wysoczynski M, Pathan A, Moore JB, Farid T, Kim J, Nasr M, et al. ProAngiogenic Actions of CMC-Derived Extracellular Vesicles Rely on Selective Packaging of Angiopoietin 1 and 2, but Not FGF-2 and VEGF. Stem Cell Rev Rep. 2019;15(4):530-42. doi: 10.1007/s12015-019-09891-6.

18. Rawat S, Gupta S, Mohanty S. Mesenchymal Stem Cells Modulate the Immune System in Developing Therapeutic Interventions. Immune Response Activation and Immunomodulation. IntechOpen; 2019. doi: 10.5772/intechopen.80772.

19. Lash GE, Pitman H, Morgan HL, Innes BA, Agwu CN, Bulmer JN. Decidual macrophages: key regulators of vascular remodeling in human pregnancy. J Leukocyte Biol. 2016;100(2):315-25. doi: 10.1189/jlb.1A0815351R.

20. Corliss BA, Azimi MS, Munson JM, Peirce SM, Murfee WL. Macrophages: An Inflammatory Link Between Angiogenesis and Lymphangiogenesis. Microcirculation. 2016;23(2):95-121. doi: 10.1111/micc.12259.

21. Wang J, Li R, Peng Z, Hu B, Rao X, Li J. HMGB1 participates in LPS-induced acute lung injury by activating the AIM2 inflammasome in macrophages and inducing polarization of M1 macrophages via TLR2, TLR4, and RAGE/NF- $\kappa$ B signaling pathways. Int J Mol Med. 2020;45:61-80. doi:10.3892/ijmm.2020.4530.

22. Fiori A, Hammes H, Bieback K. Adipose-derived mesenchymal stromal cells reverse high glucose-induced reduction of angiogenesis in human retinal microvascular endothelial cells. Cytotherapy. 2020;22(5):261-75. doi: 10.1016/j.jcyt.2020.02.005.

23. Benz PM, Ding Y, Stingl H, Loot AE, Zink J, Wittig I, et al. AKAP12 deficiency impairs VEGF-induced endothelial cell migration and sprouting. Acta Physiol. 2019;228(1). doi:10.1111/apha.13325.

24. Hofmann M, Heineke J. The Impact of Endothelial Transcription Factors in Sprouting Angiogenesis. Tumor Angiogenesis: A Key Target for Cancer Therapy. Springer; 2019. p. 73-90. doi: 10.1007/978-3-319-33673238.

25. Pertyńska-Marczewska M, Kiriakidis S, Wait R, Beech J, Feldmann M, Paleolog EM. Advanced glycation end products upregulate angiogenic and pro-inflammatory cytokine production in human monocyte/macrophages. Cytokine. 2004;28(1):35-47. doi: 10.1016/j.cyto.2004.06.006.

26. Spiller KL, Nassiri S, Witherel CE, Anfang RR, Ng J, Nakazawa KR, et al. Sequential delivery of immunomodulatory cytokines to facilitate the M1-to-M2 transition of macrophages and enhance vascularization of bone scaffolds. Biomaterials. 2015;37:194-207. doi: 10.1016/j.biomaterials.2014.10.017.

27. Moore EM, West JL. Harnessing Macrophages for Vascularization in Tissue Engineering. Ann Biomed Eng. 2018;47(2):354-65. doi: 10.1007/s10439-018-02170-4.
28. Isali I, McClellan P, Shankar E, Gupta S, Jain M, Akkus O, et al. Pd27-05 Polarization of Macrophages from Mo to M2 When Seeded Upon Genipin-Crosslinked Collagen Mesh for Treatment of Stress Urinary Incontinence. J Urology. 2020;203:e550-1. doi: 10.1097/ju.0000000000000884.05.

29. Yin $\mathrm{Y}, \mathrm{He} \mathrm{X}$, Wang J, Wu R, Xu X, Hong Y, et al. Pore sizemediated macrophage M1-to-M2 transition influences new vessel formation within the compartment of a scaffold. Appl Mater Today. 2020;18:100466. doi: 10.1016/j.apmt.2019.100466.

30. Dong Z, Fu R, Liu L, Lu F. Stromal vascular fraction (SVF) cells enhance long-term survival of autologous fat grafting through the facilitation of M2 macrophages. Cell Biol Int. 2013;37(8):855-9. doi: 10.1002/cbin.10099.

31. Cartland S, Genner S, Zahoor A, Kavurma M. Comparative Evaluation of TRAIL, FGF-2 and VEGF-A-Induced Angiogenesis In Vitro and In Vivo. Int J Mol Sci. 2016;17(12):2025. doi: 10.3390/ijms17122025.

32. Johnson KE, Wilgus TA. Vascular Endothelial Growth Factor and Angiogenesis in the Regulation of Cutaneous Wound Repair. Adv Wound Care. 2014;3(10):647-61. doi: 10.1089/wound.2013.0517.

33. Camara A, Cordeiro OG, Alloush F, Sponsel J, Chypre M, Onder L, et al. Lymph Node Mesenchymal and Endothelial Stromal Cells Cooperate via the RANK-RANKL Cytokine Axis to Shape the Sinusoidal Macrophage Niche. Immunity. 2019;50(6):1467-1481000000. doi: 10.1016/j.immuni.2019.05.008.

34. Helmke A, Casper J, Nordlohne J, David S, Haller H, Zeisberg EM, et al. Endothelial-to-mesenchymal transition shapes the atherosclerotic plaque and modulates macrophage function. FASEB J. 2018;33(2):227889. doi: 10.1096/fj.201801238R.

35. Nucera S, Biziato D, De Palma M. The interplay between macrophages and angiogenesis in development, tissue injury and regeneration. Int J Dev Biol. 2011;55(4-5):495-503. doi:10.1387/ijdb.103227sn.

36. Sunderkötter C, Steinbrink K, Goebeler M, Bhardwaj R, Sorg C. Macrophages and angiogenesis. J Leukocyte Biol. 1994;55(3):410-22. doi: 10.1002/jlb.55.3.410.

37. Lee JW, Lee S, Chun J, Im JP, Seo S, Ha N, et al. Novel Histone Deacetylase 6 Inhibitor CKD-506 Inhibits NF- $\kappa$ B Signaling in Intestinal Epithelial Cells and Macrophages and Ameliorates Acute and Chronic Murine Colitis. Inflamm Bowel Dis. 2020;26(6):852-62. doi: 10.1093/ibd/izz317.

38. Lu K, Zhao J, Liu W. Macrophage stimulating 1-induced inflammation response promotes aortic aneurysm formation through triggering endothelial cells death and activating the NF- $\kappa \mathrm{B}$ signaling pathway. J Recept Sig Transd. 2020;40(4):374-82. doi: 10.1080/10799893.2020.1738484.

39. Ji L, Zhao X, Zhang B, Kang L, Song W, Zhao B, et al. Slc6a8-Mediated Creatine Uptake and Accumulation Reprogram Macrophage Polarization via Regulating Cytokine Responses. Immunity. 2019;51(2):2722840000000. doi:10.1016/j.immuni.2019.06.007.

40. Juhas U, Ryba-Stanisławowska M, Brandt-Varma A, Myśliwiec M, Myśliwska J. Monocytes of newly diagnosed juvenile DM1 patients are prone to differentiate into regulatory IL-10+ M2 macrophages. Immunol Res. 2019;67(1):58-69. doi: 10.1007/s12026-019-09072-0.

41. Pajarinen J, Lin T, Gibon E, Kohno Y, Maruyama M, Nathan K, et al. Mesenchymal stem cell-macrophage crosstalk and bone healing. Biomaterials. 2019;196:80-9. doi: 10.1016/j.biomaterials.2017.12.025.

42. Wang Y, Chang T, Wu T, Xu W, Dou G, Wang Y, et al. M2 macrophages promote vasculogenesis during retinal neovascularization by regulating bone marrow-derived cells via SDF-1/VEGF. Cell Tissue Res. 2020;380(3):469-86. doi:10.1007/s00441-019-03166-9.

43. Ishida Y, Gao J, Murphy PM. Chemokine Receptor CX3CR1 Mediates Skin Wound Healing by Promoting Macrophage and Fibroblast Accumulation and Function. J Immunol. 2008;180(1):569-79. doi: 10.4049/jimmunol.180.1.569.

44. Bösmüller H, Pfefferle V, Bittar Z, Scheble V, Horger M, Sipos B, et al. Microvessel density and angiogenesis in primary hepatic malignancies: Differential expression of CD31 and VEGFR-2 in hepatocellu- 
lar carcinoma and intrahepatic cholangiocarcinoma. Pathol Res Pract. 2018;214(8):1136-41. doi:10.1016/j.prp.2018.06.011.

45. Richards J, Gabunia K, Kelemen SE, Kako F, Choi ET, Autieri MV. Interleukin-19 increases angiogenesis in ischemic hind limbs by direct effects on both endothelial cells and macrophage polarization.J Mol Cell Cardiol. 2015;79:21-31. doi:10.1016/j.yjmcc.2014.11.002.

46. Liu W, Wen S, Zhan J, Li Y, Shen J, Yang W, et al. Treatment with Recombinant Trichinella spiralis Cathepsin B-like Protein Ameliorates Intestinal Ischemia/Reperfusion Injury in Mice by Promoting a Switch from M1 to M2 Macrophages.JImmunol.2015;195(1):317-28. doi: 10.4049/jimmunol.1401864.

47. Lee HD, Kim YH, Kim D. Exosomes derived from human macrophages suppress endothelial cell migration by controlling integrin trafficking. Eur J Immunol. 2014;44(4):1156-69. doi: 10.1002/eji.201343660.

48. Wang C, Zhang C, Liu L, A X, Chen B, Li Y, et al. Macrophage-Derived mir-155-Containing Exosomes Suppress Fibroblast Proliferation and Promote Fibroblast Inflammation during Cardiac Injury. Mol Ther. 2017;25(1):192-204. doi: 10.1016/j.ymthe.2016.09.001.

49. Zheng B, Yin W, Suzuki T, Zhang X, Zhang Y, Song L, et al. ExosomeMediated miR-155 Transfer from Smooth Muscle Cells to Endothelial Cells Induces Endothelial Injury and Promotes Atherosclerosis. Mol Ther. 2017;25(6):1279-94. doi:10.1016/j.ymthe.2017.03.031.

50. Bardi GT, Smith MA, Hood JL. Melanoma exosomes promote mixed M1 and M2 macrophage polarization. Cytokine. 2018;105:63-72. doi: 10.1016/j.cyto.2018.02.002.

51. Fan R, Xiao C, Wan X, Cha W, Miao Y, Zhou Y, et al. Small molecules with big roles in microRNA chemical biology and microRNA-targeted therapeutics. RNA Biol. 2019;16(6):707-18. doi: 10.1080/15476286.2019.1593094.

52. Xie Y, Wang M, Tian J, Li X, Yang M, Zhang K, et al. Long non-coding RNA expressed in macrophage co-varies with the inflammatory phenotype during macrophage development and polarization.J Cell Mol Med. 2019;23(10):6530-42. doi: 10.1111/jcmm.14557.

53. Zhang Y, Zhang M, Zhong MIN, Suo Q, Lv KUN. Expression profiles of miRNAs in polarized macrophages. Int J Mol Med. 2013;31(4):797-802. doi: 10.3892/ijmm.2013.1260.

54. Newman AC, Hughes CC. Macrophages and angiogenesis: a role for Wnt signaling. Vasc Cell. 2012;4(1):13. doi:10.1186/2045-824X-4-13.

55. Bergenfelz C, Medrek C, Ekström E, Jirström K, Janols H, Wullt M, et al. Wnt5a Induces a Tolerogenic Phenotype of Macrophages in Sepsis and Breast Cancer Patients. J Immunol. 2012;188(11):5448-58. doi: 10.4049/jimmunol.1103378.

56. Kim J, Kim J, Kim DW, Ha Y, Ihm MH, Kim H, et al. Wnt5a Induces Endothelial Inflammation via $\beta$-Catenin-Independent Signaling. J Immunol. 2010;185(2):1274-82. doi: 10.4049/jimmunol.1000181.

57. Masckauchán TH, Agalliu D, Vorontchikhina M, Ahn A, Parmalee NL, Li C, et al. Wnt5a Signaling Induces Proliferation and Survival of En- dothelial Cells In Vitro and Expression of MMP-1 and Tie-2. Mol Biol Cell. 2006;17(12):5163-72. doi: 10.1091/mbc.e06-04-0320.

58. Malsin ES, Kim S, Lam AP, Gottardi CJ. Macrophages as a Source and Recipient of Wnt Signals. Front Immunol. 2019;10. doi: 10.3389/fimmu.2019.01813.

59. Abaricia JO, Shah AH, Chaubal M, Hotchkiss KM, Olivares-Navarrete R Wnt signaling modulates macrophage polarization and is regulated by biomaterial surface properties. Biomaterials. 2020;243:119920. doi: 10.1016/j.biomaterials.2020.119920.

60. Loughna S, Sato TN. Angiopoietin and Tie signaling pathways in vascular development. Matrix Biol. 2001;20(5-6):319-25. doi: 10.1016/s0945-053x(01)00149-4.

61. Engel JE, Williams E, Williams ML, Bidwell GL, Chade AR. Targeted VEGF (Vascular Endothelial Growth Factor) Therapy Induces LongTerm Renal Recovery in Chronic Kidney Disease via Macrophage Polarization. Hypertension. 2019;74(5):1113-23. doi: 10.1161/hypertensionaha.119.13469.

62. Qiu M, Ma J, Zhang J, Guo X, Liu Q, Yang Z. MicroRNA-150 deficiency accelerates intimal hyperplasia by acting as a novel regulator of macrophage polarization. Life Sci. 2020;240:116985. doi: 10.1016/j.lfs.2019.116985.

63. Bruen R, Curley S, Kajani S, Lynch G, O’Reilly ME, Dillon ET, et al Different monocyte phenotypes result in proresolving macrophages in conjugated linoleic acid-induced attenuated progression and regression of atherosclerosis. FASEB J. 2019;33(10):11006-20. doi: 10.1096/fj.201900922R.

64. Zhang S, Chen L, Zhang G, Zhang B. Umbilical cord-matrix stem cells induce the functional restoration of vascular endothelial cells and enhance skin wound healing in diabetic mice via the polarized macrophages. Stem Cell Res Ther. 2020;11(1). doi: 10.1186/s13287-020 1561-x.

65. Ganta VC, Choi M, Farber CR, Annex BH. Antiangiogenic VEGF 165 b Regulates Macrophage Polarization via S100A8/S100A9 in Peripheral Artery Disease. Circulation. 2019;139(2):226-42. doi: 10.1161/circulationaha.118.034165.

66. Kawai Y, Narita Y, Yamawaki-Ogata A, Usui A, Komori K. Montelukast, a Cysteinyl Leukotriene Receptor 1 Antagonist, Induces M2 Macrophage Polarization and Inhibits Murine Aortic Aneurysm Formation. BioMed Res Int. 2019;2019:1-11. doi: 10.1155/2019/9104680.

67. Cheng Y, Cheng T, Qu Y. TIMP-3 suppression induces choroidal neovascularization by moderating the polarization of macrophages in age-related macular degeneration. Mol Immunol. 2019;106:119-26. doi: 10.1016/j.molimm.2018.12.026.

68. Shimizu K, Kushamae M, Mizutani T, Aoki T. Intracranial Aneurysm as a Macrophage-mediated Inflammatory Disease. Neurol Medico-Chir. 2019;59(4):126-32. doi: 10.2176/nmc.st.2018-0326. 